\title{
Analytic random-walk model for the coherence of a frequency comb
}

\author{
R. Eramo, ${ }^{1,2,3}$ P. Cancio Pastor, ${ }^{1,2,3}$ and S. Cavalieri ${ }^{1,2}$ \\ ${ }^{1}$ European Laboratory for Non-Linear Spectroscopy (LENS), Via N. Carrara 1, I-50019 Sesto Fiorentino, Firenze, Italy \\ ${ }^{2}$ Dipartimento di Fisica e Astronomia, Università di Firenze, Via G. Sansone 1, I-50019 Sesto Fiorentino, Firenze, Italy \\ ${ }^{3}$ Istituto Nazionale di Ottica (INO-CNR), Largo E. Fermi 6, I-50125 Firenze, Italy
}

(Received 22 December 2017; published 21 March 2018)

\begin{abstract}
We present an analytical study of the frequency comb coherence due to random noise in the pulses phases. We derive a simple expression for the comb lineshape, which depends on a single parameter $N_{\text {eff }}$ with the physical meaning of number of coherent comb pulses, inversely proportional to the variance of the phase jumps between subsequent comb pulses. A comparison to the case of a cw-monomode laser with white noise frequency fluctuations is also presented.
\end{abstract}

DOI: 10.1103/PhysRevA.97.033842

\section{INTRODUCTION}

After the realization of the optical frequency comb (OFC) [1-5], the regular comb of spectral teeth of a mode-locked laser spectrum has been extensively used as an absolute frequency ruler $[6,7]$ or as a multiwavelength laser source for spectroscopic applications [8-13]. The ultimate frequency precision and spectral resolution in these OFC applications are determined by the tooth linewidth, which is $\Delta v \approx v_{\text {rep }} / N_{\text {eff }}$, with $v_{\text {rep }}$ being the frequency of the circulating pulse in the comb cavity, and $N_{\text {eff }}$ the number of comb pulses that are coherently involved in the measurement. Each OFC can be characterized by the value of $N_{\text {eff }}$, which is determined by the cavity-length and offset-frequency fluctuations, laser-pump intensity noise, as well as fluctuations associated with the residual noise of the electronic stabilization loop(s). Stateof-the-art fiber-based OFCs in the optical region allow a projected submillihertz comb tooth linewidths corresponding to acquisition times of the order of $10^{3} \mathrm{~s}$, and $N_{\mathrm{eff}} \sim 10^{11}$ $[14,15]$.

Recently [16], by using a properly devised interferometer [17], we experimentally investigated the pulse-to-pulse phase jitter of a fiber-based metrological frequency comb, measuring the phase-jitter power spectral distribution (PSD) for Fourier frequencies between around $1 \mathrm{MHz}$ and the Nyquist value $v_{\text {rep }} / 2$. This frequency interval is of particular interest in the field of combs in the extreme ultraviolet (XUV) spectral region, which have been recently realized by using high-order harmonic generation (HHG) from a frequency comb laser in the near infrared (NIR) [12], and whose $\mathrm{MHz}$ linewidths indicate a relatively small number of comb pulses coherently involved $\left(N_{\text {eff }} \sim 100\right)$. The experimental phase-jitter PSD shows a continuous distribution of noise extending to the Nyquist frequency, whose magnitude depends on the comb amplification. Over such distribution relatively-low-frequency monochromatic oscillations appear and contribute to the total pulse jitter phase variance. In principle, with the evolution of the electronics [18], such low-frequency oscillations could be eliminated or greatly reduced and it is thus useful to estimate the limit resolution achievable when only the flat noise floor is left, as in the quantum-like case [19-21]. ${ }^{1}$ In this paper we investigate the spectral effect in the OFC modes of a randomwalk distribution of phase noise in the comb pulses, deriving simple analytical expressions for the spectral coherence.

The paper is organized as follows: First we present a general treatment of the OFC spectrum with phase noise (Sec. II), considering both the uncorrelated and the correlated pulsephase jump cases. In Sec. III we discuss the phase-diffusion model, which corresponds to the case of a free running OFC. Finally, we conclude with a comparison between the modes of the phase-diffusion model comb and the case of $\mathrm{cw}$ monomode laser with white-noise frequency fluctuations (Sec. IV).

\section{COMB MODEL}

We start from the following expression for the electric field of a comb of $N$ pulses [22]:

$$
E(t)=\sum_{n=0}^{N-1} e^{i \psi_{n}} F\left(t-n T_{\text {rep }}\right) e^{2 \pi i v_{c} t}+\text { c.c. },
$$

where $T_{\text {rep }}=1 / \nu_{\text {rep }}$ is the comb circulation period, $v_{\mathrm{c}}=$ $v_{\text {off }}+m_{c} v_{\text {rep }}$ is the carrier frequency ( $m_{c}$ is an integer), $v_{\text {off }}=$ $\nu_{\text {rep }} \Delta \phi_{\text {ceo }} / 2 \pi$ is the offset frequency, with $\Delta \phi_{\text {ceo }}$ being the carrier-envelope phase slip. As compared with Ref. [22], we introduced a phase term $e^{i \psi_{n}}$ to allow a phase jitter between subsequent pulses; the phase difference between two subsequent pulses being $\psi_{n}-\psi_{n-1}+\Delta \phi_{\text {ceo }}$. We consider the phase jumps $\psi_{n}-\psi_{n-1}$ as $N-1$ stationary Gaussian processes and neglect the noise in the pulse amplitude by assuming a common pulse envelope $F$, the integral of $|F|^{2}$ being proportional to the pulse energy. ${ }^{2}$ For the ideal case $\psi_{n} \rightarrow 0$ and

\footnotetext{
${ }^{1}$ The phase fluctuations induced by spontaneous emission in the intracavity pulse intensity is expected to give the ultimate fundamental quantum limit with a Schawlow-Townes linewidth contribution equal for all comb modes, but other high-level quantum noise with similar pulse-phase noise behavior is present in this kind of laser source.

${ }^{2}$ We assume short pulses: $F\left(t-n T_{\text {rep }}\right) F\left(t-m T_{\text {rep }}\right) \approx 0$ for $m \neq n$.
} 
$N \rightarrow \infty$, Eq. (1) becomes $E(t)=e^{2 \pi i v_{c} t} G(t)+$ c.c., where $G(t)=\sum_{n=-\infty}^{+\infty} F\left(t-n T_{\text {rep }}\right)$ is a complex periodic function $\left[G\left(t+T_{r}\right)=G(t)\right]$, as reported in Ref. [23].

In this paper we assume the phase jumps to be independent stationary Gaussian processes to model the free-running combpulse phase behavior. Anyhow, as a phase-control and locking systems is involved in almost all OFC applications, we consider in the next discussion on comb coherence the case of correlated phase jumps.

\section{A. Comb coherence}

Let us consider an ideal experiment of interference between two comb pulses, where the comb field is transmitted through an amplitude-splitting interferometer, introducing a delay $k T_{\text {rep }}{ }^{3}$ thus giving a signal from the interference between couples of pulses $n, n+k$. Because the pulse amplitude is assumed the same for all pulses, the visibility of the interference signal is determined by the phase difference:

$$
\begin{aligned}
\Psi_{k}^{(n)} & =k \Delta \phi_{\text {ceo }}+\psi_{n+k}-\psi_{n} \\
& =k \Delta \phi_{\text {ceo }}+\sum_{h=0}^{k-1}\left(\psi_{n+h+1}-\psi_{n+h}\right) .
\end{aligned}
$$

The variance of $\Psi_{k}^{(n)}$ grows as a function of delay $k$ due to the accumulation of random-phase jumps, leading to progressive dithering of the interference fringe. For a stationary noise process we can drop the pulse index superscript and find a variance

$$
\begin{aligned}
\sigma_{k}^{2} & =\left\langle\left(\Psi_{k}^{(n)}-\left\langle\Psi_{k}^{(n)}\right\rangle\right)^{2}\right\rangle \\
& =\sum_{h, h^{\prime}=0}^{k-1}\left\langle\left(\psi_{n+h+1}-\psi_{n+h}\right)\left(\psi_{n+h^{\prime}+1}-\psi_{n+h^{\prime}}\right)\right\rangle \\
& =\sum_{h, h^{\prime}=0}^{k-1} R\left(h-h^{\prime}\right),
\end{aligned}
$$

where $R(s)=\left\langle\left(\psi_{n+s+1}-\psi_{n+s}\right)\left(\psi_{n+1}-\psi_{n}\right)\right\rangle$ is the phasejump correlation function, with $R(0)=\sigma_{1}^{2}$ being the variance of the phase difference between subsequent pulses. Finally, considering that $R(-s)=R(s)$, Eq. (3) can be cast in the form (for $k \geqslant 1$ )

$$
\begin{aligned}
\sigma_{k}^{2} & =k R(0)+2 \sum_{m>m^{\prime}} R\left(m-m^{\prime}\right) \\
& =k \sigma_{1}^{2}+2 \sum_{h=1}^{k-1}(k-h) R(h) .
\end{aligned}
$$

This equation is a general relation, indicating how the phasecorrelation function determines the variance growth. The experimental functional dependence on $k$ of $\sigma_{k}^{2}$ can be used to define the number $N_{\text {eff }}$ of laser pulses that interacts coherently through

$$
\sigma_{k=N_{\text {eff }}}^{2}=1
$$

\footnotetext{
${ }^{3}$ In Ref. [16] an experiment for $k=1$ is reported.
}

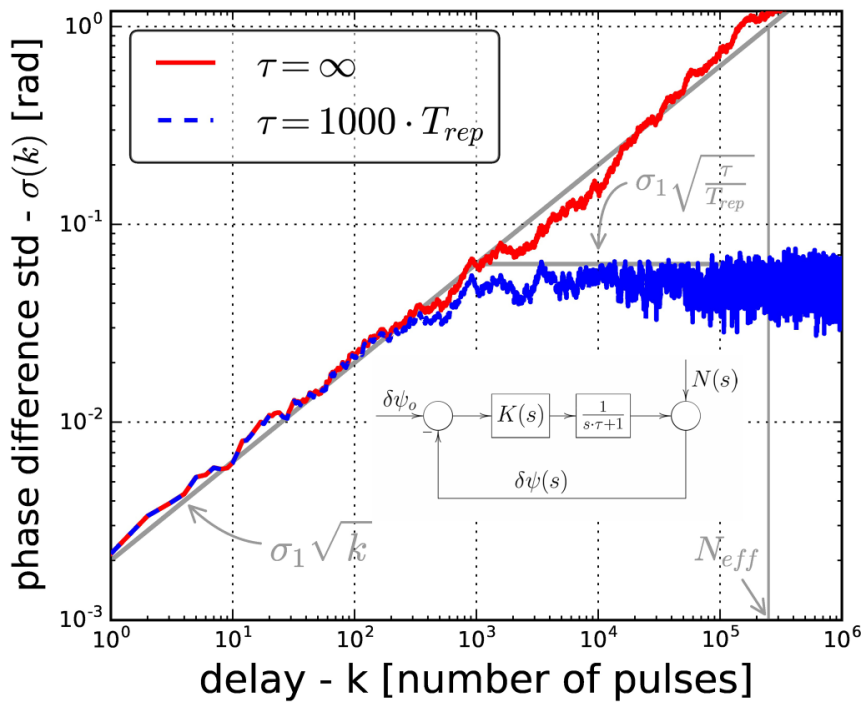

FIG. 1. Standard deviation of phase difference between $k$ pulses. Continuous red trace is the numerical simulation (average over 30 runs) where the independent phase jumps are taken from a Gaussian distribution with $\sigma_{1}=2 \mathrm{mrad}$, corresponding to the model of a free-running comb considered in this paper. Dashed blue trace shows the effect of a phase feedback loop (shown in the inset and described in the text) characterized by the response time $\tau=10^{3} T_{\text {rep }}$. The number of coherent pulses is $N_{\text {eff }}=1 / \sigma_{1}^{2}=2.5 \times 10^{5}$ for the case of no feedback, whereas it is $\infty$ in the case of the feedback loop [as for Eq. (5)]. The free-running random-walk behavior $\sigma_{k}=\sigma_{1} \sqrt{k}$, and the feedback limit $\sigma_{\infty}=\sigma_{1} \sqrt{\tau / T_{\text {rep }}}$ are also shown by light gray lines.

We will see in the following paragraphs how $N_{\text {eff }}$ determine the spectral characteristics of the comb, i.e., the contrast and the linewidth of the comb modes.

In Fig. 1 (red trace) a numerical simulation shows this growth for the case of a random extraction of independent phase jumps from a Gaussian distribution of standard deviation $\sigma_{1}=2 \mathrm{mrad}$. For independent phase jumps we have a complete loss of memory and $R(h)=\delta_{0 h} \sigma_{1}^{2}$. In this case, Eq. (4) corresponds to a linear growth of variance, $\sigma_{k}^{2}=k \sigma_{1}^{2}$, and we get $N_{\text {eff }}=1 / \sigma_{1}^{2}$.

Statistical independence cannot be assumed when some kind of active phase stabilization is operating, as shown by the saturating trace (dashed blue) of Fig. 1. We simulate this case with the system shown in the figure inset, where the phase jump is considered as a continuous variable $\delta \psi(t)$, fed to a proportional-integral controller whose Laplace response function is $K(s)=K_{P}+K_{I} / s$, with $K_{P}$ and $K_{I}$ being the proportional and integral constants, respectively. The controller drives the phase actuator, here taken in its simplest form as a first-order system characterized by a response time $\tau$ [Laplace transform $1 /(1+s \tau)$ ]. The phase noise is represented by the generator $N(s)$ (a stochastic generator) giving the interpulse phase $\delta \psi(s)$. We assume an ideally instantaneous measurement of $\delta \psi$, and the instantaneous phase error feed to the stabilization loop is the difference between the phase set ( $\delta \psi_{o}=0$ corresponds to setting a zero-phase jump between successive pulses) and the resulting jump $\delta \psi$. Considering that even the fastest controller nowadays available has a response times orders of magnitude greater than $T_{\text {rep }}$, here we report 
a simulation with $\tau=10^{3} T_{\text {rep }}$. As expected, the phase trace is stabilized in a time of the order of $\tau$ to a value depending on the loop parameters [in our example $\left.\sigma_{k}^{2} \rightarrow \sigma_{1}^{2}\left(\tau / T_{\text {rep }}\right)\right]$. In this case the correlation between phase jumps introduced by the stabilization loop breaks the linear variance growth and leads to a perfect comb $\left[N_{\text {eff }}=\infty\right.$ following our definition (5)]. In the real world, inevitable long time drifts would finally lead to a complete dephasing also for the phase-locked system: state-of-the-art systems have demonstrated coherence figures as high as $N_{\text {eff }}=10^{11}[14,15]$. Nevertheless, the initial linear growth of the stabilized system, during $\approx \tau$, is relevant in the generation of combs in the extreme ultraviolet (XUV) through high-order harmonic generation, where it determines the comb linewidth, as we pointed out in previous work [16].

\section{B. Comb spectrum}

Taking the Fourier transform of Eq. (1), we get after few simple passages

$$
\tilde{E}(\nu)=\tilde{F}\left(\nu-v_{c}\right) \sum_{n=0}^{N-1} e^{i \psi_{n}} e^{-2 \pi i n \frac{v-v_{c}}{\nu_{\text {rep }}}}+\operatorname{cc}[-v],
$$

where $\mathrm{cc}[-\nu]$ is an abbreviation for the complex conjugate of the previous term, with the replacement $v \rightarrow-v{ }^{4}$ For short pulses, of duration $\tau_{p} \sim 10 \mathrm{fs}$, the Fourier transform of the envelope, $\tilde{F}$, is a wide bump of width $\sim 1 / \tau_{p} \sim 100 \mathrm{THz}$. If we consider a range of detunings $\Delta v=v-v_{\mathrm{c}}$ around the carrier $v_{\mathrm{c}}$, with $|\Delta v| \ll 1 / \tau_{p}$, the cc term in Eq. (6) is negligible and we are led to the following expression for the field power spectrum:

$$
I_{E}(\Delta v) \propto|\tilde{E}|^{2}=|\tilde{F}(\Delta v)|^{2}\left|\sum_{n=0}^{N-1} e^{i \psi_{n}} e^{-2 \pi i n \frac{\Delta v}{v_{\text {rep }}}}\right|^{2} .
$$

For $v \rightarrow v \pm v_{\text {rep }}$ each complex exponential is unchanged: as expected, neglecting the envelope frequency dependency, the spectrum is a comb, a periodic function with period $v_{\text {rep }}$. Moreover, for $\psi_{n} \rightarrow 0$ the comb peaks correspond to the mode frequencies $v=v_{\text {off }}+m v_{\text {rep }}$ ( $m$ integer). In the following we study in some detail the comb part of Eq. (7), which in dimensionless units can be written as $I_{E}(\Delta v) \propto Z_{N}(x)$, where $x=\frac{\Delta v}{v_{\text {rep }}}$ and

$$
Z_{N}(x)=\left|\sum_{n=0}^{N-1} e^{i \psi_{n}} e^{-2 \pi i n x}\right|^{2}
$$

The average of Eq. (8) over phase fluctuations is calculated in the next paragraph. Here we conclude with a brief discussion of an ideal case, labeled by the superscript ${ }^{(i d)}$, to be used as reference in the following: a comb with finite number of pulses and no phase jitter $\left(\psi_{n}=0\right)$. To determine simple analytical expressions for the lineshape, here we consider the frequency interval around a maximum of the spectrum, i.e., a comb peak.

\footnotetext{
${ }^{4}$ As Fourier transform of a real function, the field spectrum is always of the form $\tilde{E}(v)=f(v)+f(-v)^{*}$, where $f$ is a complex function of $v$. This ensures that $\tilde{E}(-v)=\tilde{E}(v)^{*}$.
}

Elementary algebra shows that

$$
\begin{aligned}
Z_{N}^{(i d)}(x) & =\left|\frac{1-e^{-2 \pi i x N}}{1-e^{-2 \pi i x}}\right|_{x=\frac{\Delta v}{v_{\text {rep }}}}^{2}=N^{2}\left|\frac{\sin (\pi x N)}{N \sin (\pi x)}\right|_{x=\frac{\Delta v}{v_{\text {rep }}}}^{2} \\
& \approx N^{2} \operatorname{sinc}^{2}(N x) .
\end{aligned}
$$

Thus, in this limit the modes are sinc shaped, ${ }^{5}$ i.e., they have a diffraction-type lineshape, and mode contrast ${ }^{6}$ and mode linewidth (FWHM) are respectively given by

$$
\begin{gathered}
\rho_{N}^{(i d)}=N^{2}, \\
\Delta v_{N}^{(i d)}=v_{\text {rep }} \frac{0.886}{N} .
\end{gathered}
$$

\section{PHASE-DIFFUSION MODEL}

In this section we consider a phase-diffusion model where the phase jumps are independent stationary Gaussian processes, with mean value $\mu=0$, and with a common standard deviation $\sigma_{1}$.

We note that the dimensionless spectrum (8) for $e^{-2 \pi i n x} \rightarrow$ 1 is the expression of the $N$ th step position in a random walk of a particle in the plane, this being the case of a unitary freeflight length between two collisions, and $\psi_{n}$ being the emerging angle after a collision. Different from the plane random walk where a flat emission probability covers an angle of $2 \pi$, in the comb case the angle is in general restricted to a small region around the origin, which can be quantified by the standard deviation $\sigma_{1}$ of the comb phase diffusion. In the averaging of Eq. (8),

$$
\left\langle Z_{N}(x)\right\rangle=N+\left\langle\sum_{n>m} e^{i\left(\psi_{n}-\psi_{m}\right)} e^{-2 \pi i(n-m) x}+\text { c.c. }\right\rangle
$$

let us consider a term with $n>m$ as a succession of phase jumps between subsequent pulses, and remember that they are statistically uncorrelated:

$$
\begin{aligned}
& \left\langle e^{i\left(\psi_{n}-\psi_{m}\right)} e^{-2 \pi i(n-m) x}+\text { c.c. }\right\rangle \\
& =\underbrace{\left\langle e^{i\left(\psi_{n}-\psi_{n-1}\right)}\right\rangle \cdots\left\langle e^{i\left(\psi_{m+1}-\psi_{m}\right)}\right\rangle}_{\mathrm{n}-\mathrm{m} \text { terms }} e^{-2 \pi i(n-m) x}+\text { c.c. } \\
& =e^{-(n-m) \sigma_{1}^{2} / 2} e^{-2 \pi i(n-m) x}+\text { c.c. } \\
& =2 e^{-(n-m) \sigma_{1}^{2} / 2} \cos 2 \pi(n-m) x,
\end{aligned}
$$

having considered that, for a stochastic variable $y_{g}$ with a normal distribution of mean value $\mu_{g}$ and standard deviation

\footnotetext{
${ }^{5} Z_{N}(x) \approx N^{2} \operatorname{sinc}^{2}(x N)$ for $|x| \ll 1$.

${ }^{6}$ The mode contrast is defined as the ratio between the peack value $I_{o}$ and the plateau level in the intermediate region between two successive peaks. To evaluate it, we note that the denominator of Eq. (9) has unit period when $x=1 / 2+\delta x$, and it is nearly at its maximum, while the numerator has period $1 / N$ and zeros in $\delta x= \pm 1 / 2 N$ ( $N$ odd $)$, or in $\delta x=0$ ( $N$ even $)$. Thus, the local maxima of the numerator in the plateau region are in $\delta x= \pm 1 / 2 N$ ( $N$ even) or in $\delta x=0$ ( $N$ odd). Taking the minimum of the maxima as the plateau, $Z_{N} \approx 1$, and consequently the contrast is $N^{2}$.
} 

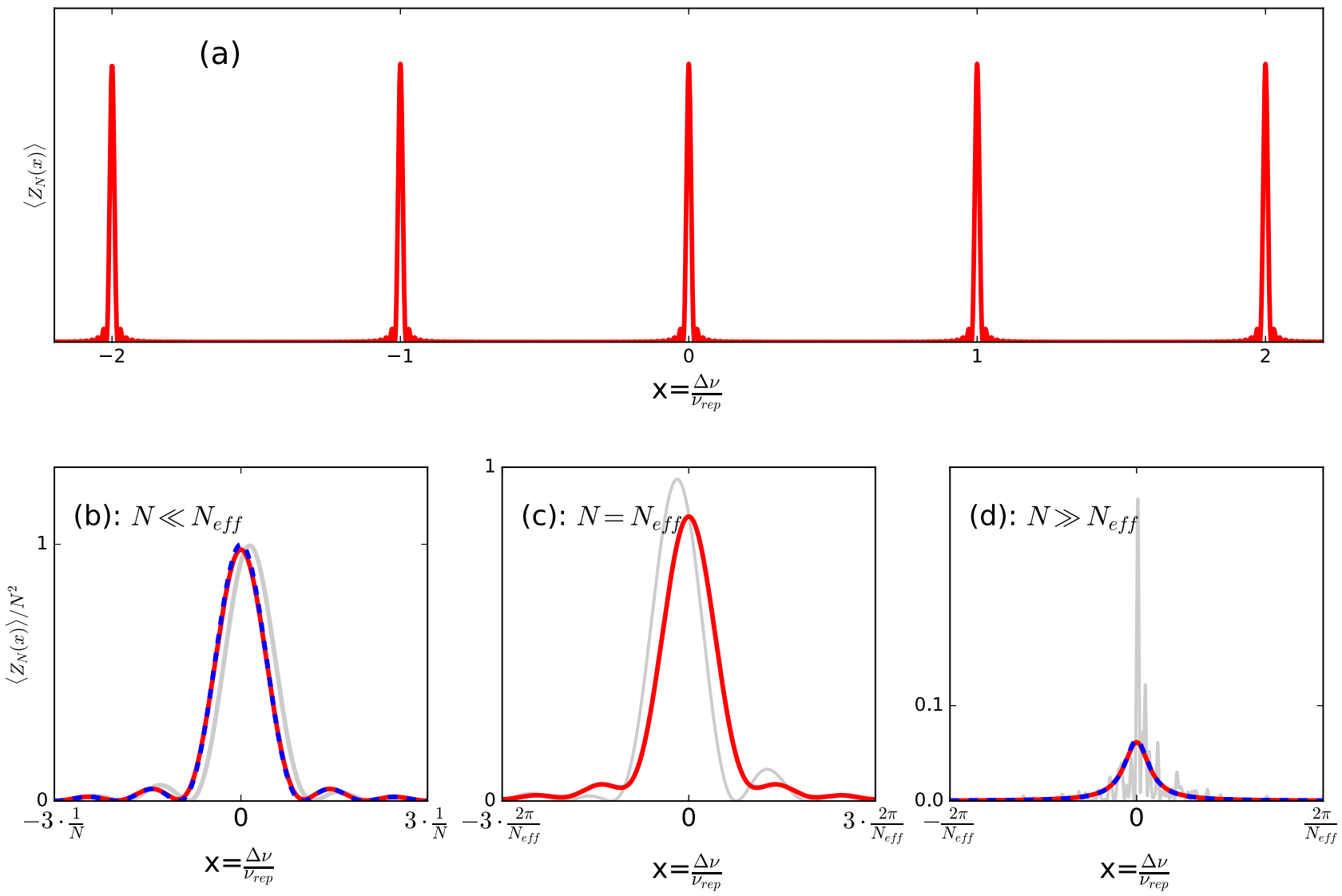

FIG. 2. Comb-field intensity spectrum $Z_{N}$ [Eq. (8)] as a function of the normalized frequency detuning $x=\Delta v / v_{\text {rep }}$ (distance from the carrier frequency measured in units of $v_{\text {rep }}$ ). (a) Ideal case (no phase fluctuations) $Z_{N}(x)=\sin ^{2}(\pi N x) / \sin ^{2}(\pi x)$; the comb modes appear on integers of $x$. The dimensionless comb-field intensity spectrum normalized to $N^{2}$ for one comb mode is shown for the three regimes:

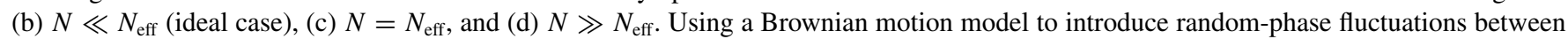
successive comb pulses, the spectrum [gray lines in panels (b)-(d)] presents an irregular shape for $N \geqslant N_{\text {eff }}$. Averaging over such spectra leads to an analytic expression, shown by red lines, whose shape depends on the number of coherent pulses $N_{\text {eff }}=1 / \sigma^{2}$. Around the integers the spectrum is well approximated by a sinc shape in panel (b) and by the Lorentzian curve (with $\Gamma=1 / 2 \pi N_{\text {eff }}$ as FWHM) in panel (d). These two asymptotic curves are plotted as dashed lines. Note the different scales in panels (a)-(c) $\left(1 / N\right.$ vs $\left.2 \pi / N_{\text {eff }}\right)$.

$\sigma_{g}$, is $\left\langle e^{i y_{g}}\right\rangle=e^{i \mu_{g}-\sigma_{g}^{2} / 2}$. Thus, averaging over the phase jumps leads to the following expression of the comb spectrum:

$$
\left\langle Z_{N}(x)\right\rangle=N+2 \sum_{n>m} e^{-(n-m) \sigma_{1}^{2} / 2} \cos 2 \pi(n-m) x,
$$

and, considering that there are $N-1$ terms with $n-m=1$, $N-2$ terms with $n-m=2, \ldots$, and that there is just one term with $n-m=N-1$ :

$$
\begin{aligned}
\left\langle Z_{N}(x)\right\rangle & =N+2 \sum_{k=1}^{N-1}(N-k) e^{-k \sigma_{1}^{2} / 2} \cos 2 \pi k x \\
& =N+\sum_{k=1}^{N-1}(N-k)\left[u^{k}+\text { c.c. }\right],
\end{aligned}
$$

where the following notations are used:

$$
\begin{gathered}
u=e^{\alpha}, \\
\alpha=2 \pi(i x-\Gamma / 2), \\
\Gamma=\sigma_{1}^{2} / 2 \pi=\frac{1}{2 \pi N_{\mathrm{eff}}} .
\end{gathered}
$$

The sum can be calculated in closed form by using a property of the geometric series, ${ }^{7}$ obtaining finally

$$
\begin{aligned}
\left\langle Z_{N}(x)\right\rangle= & N+\left[N\left(-1+\frac{1-u^{N}}{1-u}\right)+\frac{N u^{N}}{1-u}\right. \\
& \left.-u \frac{1-u^{N}}{(1-u)^{2}}+\text { c.c. }\right] .
\end{aligned}
$$

A key parameter in this expression of the comb spectrum is the number $N_{\text {eff }}$ of coherent pulses, which defines two regimes: $N / N_{\text {eff }}$ is $\gg 1$ or $\ll 1$. The spectroscopic characteristics of the comb in these two regimes are graphically summarized in Fig. 2, where the average spectrum and a simulated spectrum are compared for three cases : $N \ll N_{\text {eff }}$ [Fig. 2(b)], $N \sim N_{\text {eff }}$ [Fig. 2(c)], and $N \gg N_{\text {eff }}$ [Fig. 2(d)]. Mode linewidth and contrast behavior are illustrated in Fig. 3.

${ }^{7}$ We start from the geometric series formula $\sum_{k=0}^{N-1} u^{k}=\frac{1-u^{N}}{1-u}$. Differentiating with respect to $u$, and multiplying by $u$, we obtain $\sum_{k=0}^{N-1} k u^{k}=\frac{-N u^{N}}{1-u}+u \frac{1-u^{N}}{(1-u)^{2}}$. From this we obtain $\sum_{k=1}^{N-1}(N-$ k) $u^{k}=N\left(-1+\frac{1-u^{N}}{1-u}\right)+\frac{N u^{N}}{1-u}-u \frac{1-u^{N}}{(1-u)^{2}}$. 

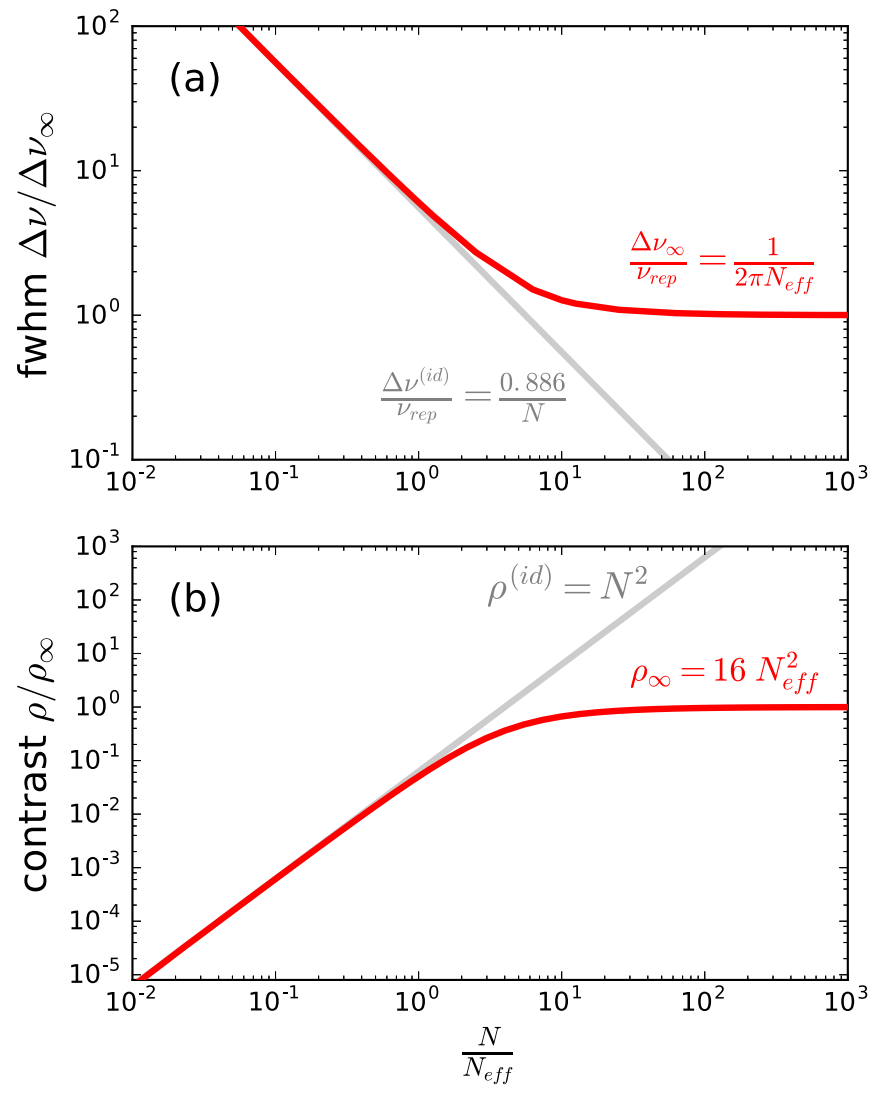

FIG. 3. In panels (a) and (b) the linewidth $\Delta v$ (the FWHM of the mode peak), and the contrast $\rho$ (peak value over the plateau level of the spectrum), normalized by the corresponding asymptotic values for $N \rightarrow \infty\left(\Delta v_{\infty}\right.$ and $\left.\rho_{\infty}\right)$, are shown as a function of $N / N_{\text {eff. The }}$ corresponding behaviors for the ideal case (no phase fluctuations) are shown as gray lines.

For $N \ll N_{\text {eff }}$ a straightforward but lengthly calculation shows that the ideal expression limit, Eq. (9), is indeed obtained from Eq. (16). In this case each mode has a sinc lineshape, as in Fig. 2(b), with a linewidth decreasing as $1 / N$ [corresponding to the gray line of Fig. 3(a)], and a contrast increasing as $N^{2}$ [Fig. 3(b), gray line]. This is the expected result when there is no phase fluctuation in the pulses, and consequently the comb resolution increases with the number of measured comb pulses.

For $N \geqslant 2 \pi N_{\text {eff }}$ we find around each integer $k, \frac{\Delta v}{v_{\text {rep }}}=k+$ $x$, with $|x| \ll 0.5$ :

$$
\begin{aligned}
\left\langle Z_{N}(x)\right\rangle \approx & N \frac{\Gamma}{2 \pi} \frac{1}{x^{2}+\frac{\Gamma^{2}}{4}} \\
& +\frac{1}{2 \pi^{2}} \frac{x^{2}-\frac{\Gamma^{2}}{4}}{\left[x^{2}-\frac{\Gamma^{2}}{4}\right]^{2}+x^{2} \Gamma^{2}} .
\end{aligned}
$$

The first term is a Lorentzian line, with FWHM $\Gamma=1 / 2 \pi N_{\text {eff }}$ and a peak value $4 N N_{\text {eff. The second term is a dumped }}$ harmonic-oscillator term, a negative correction to the peak value which is significant for $N \simeq 2 \pi N_{\text {eff }}$ and becomes negligible for $N \rightarrow \infty$. Thus, for $N \gg N_{\text {eff }}$ the mode strength and the mode linewidth are

$$
\begin{gathered}
I_{\infty}=4 N N_{\mathrm{eff}}, \\
\frac{\Delta v_{\infty}}{v_{\mathrm{rep}}}=\frac{1}{2 \pi N_{\mathrm{eff}}} .
\end{gathered}
$$

Finally, to get the contrast in this case, the following expression can be derived for the frequency range between two successive modes:

$$
\left\langle Z_{N}(x)\right\rangle \approx N \frac{1-e^{-2 \pi \Gamma}}{1-e^{-2 \pi \Gamma}-2 e^{-\pi \Gamma} \cos (2 \pi x)} .
$$

From this we obtain a plateau value $\frac{N}{4 N_{\text {eff }}}$, i.e., an asymptotic contrast ratio

$$
\rho_{\infty}=16 N_{\text {eff }}^{2}
$$

Comparing Eqs. (21) and (19) with Eqs. (10) and (11), we find that the number $N_{\text {eff }}$ of coherent pulses, apart from a factor (which is four for the contrast, and $\sim 2 \pi$ for the width), plays the same role as $N$ in an ideal comb. From a spectroscopic point of view it is thus useless to go into the regime $N \gg N_{\text {eff }}$ : as shown in the Figs. 3(a) and 3(b), there is no advantage both in the frequency resolution and in the mode contrast. This is clearly explained by observing the gray simulation in Fig 2(d): each observation (8) made on $N$ comb pulses substantially differs from the average (17), given a constant contrast. In other words, it is not possible to gain knowledge on the mean mode frequency with an accuracy less than $\approx \frac{1}{N_{\text {eff }}}$ for a free-running OFC.

\section{COMPARISON TO A CONTINUOUS-WAVE MONOMODE LASER}

In the previous section we obtained a Lorentzian lineshape of the comb modes for large $N$. Here we consider an heuristic alternative approach illustrating the parallelism with a cw laser with white-noise frequency fluctuations. In fact, for the case of large $N$ the sum in Eq. (7) can be written as a Fourier integral. We have for $v$ around the frequency $v_{m}=v_{\text {off }}+m v_{\text {rep }}$ :

$$
\begin{aligned}
Z_{N} & =\left|\sum_{n=0}^{N-1} e^{i \psi_{n}} e^{-2 \pi i n T_{\text {rep }}\left(\nu-v_{m}\right)}\right|^{2} \\
& \approx\left|\frac{1}{T_{\text {rep }}} \int_{0}^{N T_{\text {rep }}} d t e^{-2 \pi i t v} e^{2 \pi i v_{m} t+i \psi(t)}\right|^{2} .
\end{aligned}
$$

Any mode index leads to the same spectral profile, because $v_{m}-v_{c}$ is an integer multiple of $v_{\text {rep }}$, the periodicity of the summed exponentials. The integral expression can be read as the spectrum of a quasimonochromatic field $E_{m}(t)=\cos \left[2 \pi v_{m} t+\psi(t)\right]$, where the mode phase function $\psi\left(n T_{\text {rep }}\right)=\psi_{n}$, sampled at a rate $\nu_{\text {rep }}$, is associated with an offset-frequency fluctuation

$$
\begin{aligned}
\delta \nu\left(n T_{\text {rep }}\right) & =\left(\psi_{n}-\psi_{n-1}\right) / 2 \pi T_{\text {rep }} \\
& \sim \dot{\psi}\left(n T_{\text {rep }}\right) / 2 \pi
\end{aligned}
$$

that can also be read as a mode frequency fluctuation of the underlying modes. Thus, interpulse phase jitter and equivalent mode-frequency fluctuation are indeed proportional, and the comb phase jitter is equivalent to a mode field which is a phasemodulated sinusoid.

Assuming that $\delta v$ can be represented by a stochastic stationary Gaussian process, then the average of Eq. (22) can be computed by well-known standard techniques [24,25], because $\psi(t)=2 \pi \int_{0}^{t} d t^{\prime} \delta v\left(t^{\prime}\right)$ is also a Gaussian random variable, 
with vanishing mean and variance

$$
\left\langle\psi(t)^{2}\right\rangle=4 \pi^{2} \int_{-\infty}^{+\infty} d f S_{\delta v}(f) \operatorname{sinc}^{2}(f t),
$$

where $\operatorname{sinc}(x)=\sin (\pi x) / \pi x$, and $S_{\delta v}$ is the power spectral distribution of $\delta v$. Once the latter is specified, Eq. (24) allows the calculation of the average of $\langle\tilde{E}(v)\rangle$ as the characteristic function of a Gaussian process: ${ }^{8}$

$$
\begin{aligned}
\langle\tilde{E}(v)\rangle & \propto \int_{0}^{N T_{\text {rep }}} d t e^{-2 \pi i t v} e^{2 \pi i v_{m} t}\left\langle e^{i \psi(t)}\right\rangle \\
& =\int_{0}^{N T_{\text {rep }}} d t e^{-2 \pi i t v} e^{2 \pi i v_{m} t-\left\langle\psi(t)^{2}\right\rangle / 2} .
\end{aligned}
$$

For white-noise fluctuations, i.e., $S_{\delta v}=S_{o}=$ const., we get from Eq. (24)

$$
\begin{aligned}
\left\langle\psi(t)^{2}\right\rangle & =4 \pi^{2} S_{o} \int_{-\infty}^{+\infty} d f \operatorname{sinc}^{2}(f t) \\
& =4 \pi^{2} S_{o} t=2 \pi \Gamma_{o} t
\end{aligned}
$$

provided that the product time Nyquist cutoff frequency $v_{N}=$ $v_{\text {rep }} / 2$ satisfies $t v_{N} \gg 1$. To get the rate constant $\Gamma_{o}$ in term of the phase-jitter variance $\sigma_{1}^{2}=\left\langle\left(\psi_{n}-\psi_{n-1}\right)^{2}\right\rangle$ we use Eq. (23): $2 v_{N} S_{o}=\left\langle\delta v^{2}\right\rangle=\sigma_{1}^{2} v_{\text {rep }}^{2} / 4 \pi^{2}$, giving $S_{o}=\sigma_{1}^{2} v_{\text {rep }} / 4 \pi^{2}$ and

$$
2 \pi \Gamma_{o}=\sigma_{1}^{2} v_{\text {rep }}=\frac{v_{\text {rep }}}{N_{\text {eff }}},
$$

in agreement with the dimensionless counterpart (15). Averaging of Eq. (22) leads finally to

$$
\begin{aligned}
\langle\tilde{E}(v)\rangle & \propto \int_{0}^{N T_{\mathrm{rep}}} d t e^{-2 \pi i t v} e^{2 \pi i v_{m} t-2 \pi \Gamma_{o} t / 2}+\mathrm{cc}[-v] \\
& \approx \frac{-1}{2 \pi i\left(v_{m}-v\right)-2 \pi \Gamma_{o} / 2}+\mathrm{cc}[-v],
\end{aligned}
$$

${ }^{8}$ I.e. $\langle\exp [i \psi(t)]\rangle=\exp \left(-\left\langle\psi(t)^{2}\right\rangle / 2\right)$. where the upper-limit contribution, proportional to $e^{-\pi \Gamma_{o} N T_{\text {rep }}}=e^{-N \sigma_{1}^{2} / 2}$, has been neglected. The square of Eq. (28) thus leads to Lorentzian modes, with $\Gamma_{o}$ as mode linewidth (FWHM), proportional to the variance of the phase jitter, i.e., inversely proportional to the coherence number $N_{\text {eff }}$. Each mode of the combs behaves as a cw monomode laser field with a frequency fluctuations characterized by a white-noise power spectral density [19-21].

\section{CONCLUSIONS}

We studied the comb spectrum in the presence of random jitter for the phases of the mode-locked laser pulses. On the average of multiple laser round trips, a simple analytical expression for the comb lineshape is derived which depends on a single parameter $N_{\text {eff }}$ that is inversely proportional to the variance of the phase jumps between subsequent comb pulses, and with the physical meaning of the number of coherent comb pulses. Different spectral regimes of the resulting OFC modes are analyzed as a function of the number of interacting pulses compared with the coherence number $N_{\text {eff }}$.

For interaction times shorter than the coherence time $N_{\text {eff }} T_{\text {rep }}$, the comb modes are those of a pulsed mode-locked oscillator without phase fluctuations, with increasing resolution and contrast as a function of interaction time. Although this regime is not often used for metrological comb-based applications, it turns out to be relevant to applications based on the interaction of a few laser pulses, such as the frequency comb generation in the XUV through high-order harmonic generation and its spectroscopic applications [16].

For interaction times around $N_{\text {eff }} T_{\text {rep }}$, pulses begin to be not coherently added because of the phase noise accumulated in the intermediate laser pulses. Further increase of the interaction time is of no advantage, both for the comb-mode resolution and its contrast.

Finally, we have shown that the Lorentzian mode shape resulting from our model for very long interaction times is in agreement with that expected for a cw monomode laser when limited by a white frequency noise.
[1] S. A. Diddams, D. J. Jones, J. Ye, S. T. Cundiff, J. L. Hall, J. K. Ranka, R. S. Windeler, R. Holzwarth, Th. Udem, and T. W. Hänsch, Direct Link between Microwave and Optical Frequencies with a $300 \mathrm{THz}$ Femtosecond Laser Comb, Phys. Rev. Lett. 84, 5102 (2000).

[2] Th. Udem, R. Holzwarth, and T. W. Hansch, Nobel lecture: Passion for precision, Nature (London) 416, 233 (2002).

[3] J. L. Hall, Nobel lecture: Defining and measuring optical frequencies, Rev. Mod. Phys. 78, 1279 (2006).

[4] T. W. Hänsch, Nobel lecture: Passion for precision, Rev. Mod. Phys. 78, 1297 (2006).

[5] P. Maddaloni, P. Cancio, and P. De Natale, Optical comb generators for laser frequency measurement, Meas. Sci. Technol. 20, 052001 (2009).

[6] S. A. Diddams, The evolving optical frequency comb, J. Opt. Soc. Am. B 27, B51 (2010).
[7] N. R. Newbury, Searching for applications with a fine-tooth comb, Nat. Photonics 5, 186 (2011).

[8] C. Gohle, B. Stein, A. Schliesser, Th. Udem, and T. W. Hänsch, Frequency Comb Vernier Spectroscopy for Broadband, High-Resolution, High-Sensitivity Absorption and Dispersion Spectra, Phys. Rev. Lett. 99, 263902 (2007).

[9] S. A. Diddams, L. Hollberg, and V. Mbele, Molecular fingerprinting with the resolved modes of a femtosecond laser frequency comb, Nature (London) 445, 627 (2007).

[10] B. Bernhardt, A. Ozawa, P. Jacquet, M. Jacquey, Y. Kobayashi, Th. Udem, R. Holzwarth, G. Guelachvili, T. W. Hansch, and N. Picque, Cavity-enhanced dual-comb spectroscopy, Nat. Photonics 4, 55 (2010).

[11] A. Foltynowicz, T. Ban, P. Masłowski, F. Adler, and J. Ye, Quantum-Noise-Limited Optical Frequency Comb Spectroscopy, Phys. Rev. Lett. 107, 233002 (2011). 
[12] A. Cingöz, D. C. Yost, Th. K. Allison, A. Ruehl, M. E. Fermann, I. Hartl, and J. Ye, Direct frequency comb spectroscopy in the extreme ultraviolet, Nature (London) 482, 68 (2012).

[13] M. Siciliani de Cumis, R. Eramo, N. Coluccelli, M. Cassinerio, G. Galzerano, P. Laporta, P. De Natale, and P. Cancio Pastor, Tracing part-per-billion line shifts with direct-frequency-comb vernier spectroscopy, Phys. Rev. A 91, 012505 (2015).

[14] T. R. Schibli, I. Hartl, D. C. Yost, M. J. Martin, A. Marcinkevicius, M. E. Fermann, and J. Ye, Optical frequency comb with submillihertz linewidth and more than $10 \mathrm{~W}$ average power, Nat. Photonics 2, 355 (2008).

[15] C. Benko, A. Ruehl, M. J. Martin, K. S. E. Eikema, M. E. Fermann, I. Hartl, and J. Ye, Full phase stabilization of a Yb:fiber femtosecond frequency comb via high-bandwidth transducers, Opt. Lett. 37, 2196 (2012).

[16] C. Corsi, I. Liontos, M. Bellini, S. Cavalieri, P. Cancio Pastor, M. Siciliani de Cumis, and R. Eramo, Ultimate Limit in the Spectral Resolution of Extreme Ultraviolet Frequency Combs, Phys. Rev. Lett. 118, 143201 (2017).

[17] C. Corsi, I. Liontos, S. Cavalieri, M. Bellini, G. Venturi, and R. Eramo, An ultrastable Michelson interferometer for highresolution spectroscopy in the XUV, Opt. Express 23, 4106 (2015).
[18] C.-C. Lee, C. Mohr, J. Bethge, S. Suzuki, M. E. Fermann, I. Hartl, and T. R. Schibli, Frequency comb stabilization with bandwidth beyond the limit of gain lifetime by an intracavity graphene electro-optic modulator, Opt. Lett. 37, 3084 (2012).

[19] R. Paschotta, Noise of mode-locked lasers (Parts 1 and 2), Appl. Phys. B: Lasers Opt. 79, 153 (2004).

[20] R. Paschotta, A. Schlatter, S. C. Zeller, H. R. Telle, and U. Keller, Optical phase noise and carrier-envelope offset noise of modelocked lasers, Appl. Phys. B: Lasers Opt. 82, 265 (2006).

[21] N. R. Newbury and W. C. Swann, Low-noise fiber-laser frequency combs (invited), J. Opt. Soc. Am. B 24, 1756 (2007).

[22] N. R. Newbury, I. Coddington, and W. Swann, Sensitivity of coherent dual-comb spectroscopy, Opt. Express 18, 7929 (2010).

[23] J. Reichert, R. Holzwarth, Th. Udem, and T. W. Hänsch, Measuring the frequency of light with mode-locked lasers, Opt. Commun. 172, 59 (1999).

[24] H. E. Rowe, Signals and Noise in Communication Systems (Van Nostrand Reinhold, New York, 1965).

[25] G. Di Domenico, S. Schilt, and P. Thomann, Simple approach to the relation between laser frequency noise and laser line shape, Appl. Opt. 49, 4801 (2010). 